\title{
Direct Root Penetration and Rhizome Vascular Colonization by Fusarium oxysporum f. sp. cubense are the Key Steps in the Successful Infection of Brazil Cavendish
}

Chunqiang Li, Hainan University, Haikou, China; Institute of Tropical Bioscience and Biotechnology, Chinese Academy of Tropical Agricultural Sciences, Haikou, China; and Key Laboratory of Biology and Genetic Resources of Tropical Crops, Ministry of Agriculture, China; and Jinghao Yang, Wenbin Li, Jianbo Sun, and Ming Peng, ${ }^{\dagger}$ Institute of Tropical Bioscience and Biotechnology, Chinese Academy of Tropical Agricultural Sciences and Key Laboratory of Biology and Genetic Resources of Tropical Crops, Ministry of Agriculture

\begin{abstract}
Fusarium wilt of banana, which is caused by Fusarium oxysporum f. sp. cubense, is one of the most serious diseases of banana. F. oxysporum f. sp. cubense race 1 (Foc1) and race 4 (Foc4) are the most prevalent pathogens of banana cultivars in the world. To understand the differences in the infection processes between Foc1 and Foc4, green fluorescent protein-tagged strains of $F$. oxysporum f. sp. cubense tropical race 4 (FocTR4) and Foc1 were used to inoculate 'Brazil Cavendish' banana. At 2 days postinoculation (dpi), it was observed that the spores and hyphae of both Foc1 and Foc4 attached to the root hairs and root epidermis. At 3 dpi, the hyphae of both Foc1 and Foc 4 were found in the vascular tissues of roots. However, Foc 4 was observed in the parenchymal cells of banana root, whereas Foc1 was not found

in parenchymal cells at 7 dpi. Furthermore, few Foc1 hyphae were observed in a few xylems whereas many more Foc4 hyphae were present in many xylems and phloems. Foc4 was observed in the vascular tissues of banana rhizomes, whereas no Foc1 was found in rhizomes 2 months after inoculation. The attachment process in $F$. oxysporum $\mathrm{f}$. sp. cubense infection was further studied with scanning electron microscopy. Foc4 was observed to penetrate into banana roots from the intercellular space of the epidermis and wounds, whereas Foc1 mainly penetrated from the wounds but not from the intercellular space of the epidermis. Therefore, direct root penetration and rhizome vascular colonization by $F$. oxysporum $\mathrm{f}$. sp. cubense are the key steps in the successful infection of Brazil Cavendish.
\end{abstract}

Fusarium wilt of banana (Musa spp.), also called Panama disease, is caused by the soilborne fungus Fusarium oxysporum f. sp. cubense and is a serious fungal disease in banana that limits banana production worldwide (Getha and Vikineswary 2002). Because its saprophyte can survive for numerous years in soil, $F$. oxysporum $\mathrm{f}$. sp. cubense is not easily eliminated once it is established in soil (Kurtz and Schouten 2009).

$F$. oxysporum f. sp. cubense is a soilborne pathogen that can invade the xylem tissues of roots and spread through the vascular system of pseudostems. However, it is difficult to monitor the infection process, and the first signs of infection, such as leaf discoloration or wilting, appear several weeks to several months after infection $(\mathrm{Li}$ et al. 2013). F. oxysporum f. sp. cubense comprises four races based on host susceptibility. Three races cause banana disease. Race 1 causes disease in dessert banana cultivars such as 'Gros Michel' (Musa spp., AAA group), race 2 (Foc2) affects cooking banana cultivars such as 'Bluggoe' (Musa spp., ABB group), and race 4 (Foc4) attacks 'Cavendish' banana cultivars (Musa spp., AAA group) and banana cultivars susceptible to Foc1 and Foc2 (Ploetz and Pegg 2000). Foc1 caused the collapse of the global Gros Michel trade industry in the mid-20th century (Stover 1962). The industry recovered by substituting Cavendish cultivars for Gros Michel but the new race (Foc4) is now threatening Cavendish production and causes considerable losses of Cavendish banana fruit in subtropical and tropical areas (Hwang and Ko 2004; Pearce 2003; Ploetz and Pegg 2000).

Banana is a tall, monocotyledonous plant. A large majority of the cultivated sweet banana and cooking banana cultivars are seedless triploid varieties $(2 \mathrm{n}=3 \mathrm{x}=33)$ that are sterile but develop parthenocarpic fruit (Heslop-Harrison and Schwarzacher 2007; Simmonds and Sheperd 1955). It is difficult to develop resistant cultivars in a short period. Therefore, it is important to understand the mechanism of pathogenesis, which may lead to the identification of efficient ways to control the Fusarium wilt caused by Foc4.

${ }^{\dagger}$ Corresponding author: M. Peng; E-mail: pengming@itbb.org.cn

Accepted for publication 17 July 2017.

C 2017 The American Phytopathological Society
Some studies have reported that $F$. oxysporum f. sp. cubense can penetrate into susceptible banana roots, move up through the vascular bundle, and finally cause wilt in plants (Beckman et al. 1961; Li et al. 2011; Wardlaw 1930; Xiao et al. 2013). However, little is known about the differences in the early infection processes between Foc1 and Foc4, especially the $F$. oxysporum $\mathrm{f}$. sp. cubense movement in the vascular bundle of the banana plant. And there are still have two problems we don't understand. One is why some Cavendish cultivars (such as 'Brazil') are resistant to Foc1 but susceptible to Foc4. The other is defining the difference in the infection of Brazil Cavendish between Foc1 and Foc4. Here, we compared the differences in the infection of Cavendish banana roots by Foc1 and Foc4 using a green fluorescent protein (GFP) reporter and scanning electron microscopy (SEM), mainly focusing on $F$. oxysporum f. sp. cubense movement in the vascular bundle and penetration into the root surface. We believe that better understanding of the differences in the infection processes between Foc1 and Foc4 is conducive to developing control methods for banana Fusarium wilt.

\section{Materials and Methods}

Fusarium strains and GFP tag. The F. oxysporum f. sp. cubense strains used in this study are tropical race 4 (FocTR4, a member of Foc4 that was isolated in the Hainan Island tropical area in China) and Foc1, which was isolated from Hainan Island in China (Li et al. 2013). These strains were transformed with the pCT74 vector carrying serin green fluorescent protein (sGFP) (Lorang et al. 2001). Protoplasts of Foc4 and Foc1 were transformed using a polyethylene glycol/ $\mathrm{CaCl}_{2}$-mediated transformation method as described previously (Huang et al. 2014). The growth characteristics and pathogenicity of the GFP-transformed lines were examined as described previously ( $\mathrm{Li}$ et al. 2007). To monitor the infection process, the GFP-expressing Foc4 and Foc1 strains with similar fluorescence intensities and morphological features (growth rate, spores, or hyphae) and banana plant pathogenicity similar to those of their respective wild strains were used to inoculate the banana roots and fluorescence microscopic observation ( $\mathrm{Li}$ et al. 2013). For the SEM experiment, the wild strains were used.

Pathogen preparation and banana inoculation. Pathogenic F. oxysporum f. sp. cubense was cultured in $500 \mathrm{ml}$ of potato dextrose broth (PDB) liquid medium and incubated at $28^{\circ} \mathrm{C}$ for 5 days in an incubator shaker rotating at $180 \mathrm{rpm}$. Then, spores were separated from the mycelia by filtering using sterile cheesecloth and adjusted to a final 
concentration of $10^{6}$ spores $\mathrm{ml}^{-1}$. The Brazil banana cultivar of the Cavendish subgroup contains the Musa AAA genome and was used in this study; this cultivar is highly susceptible to Foc4 but resistant or tolerant to Foc1. Banana plantlets were grown in pots for 2 months at $30^{\circ} \mathrm{C}$, approximately $85 \%$ relative humidity, and a 12 - to 13 -h cycle of light and darkness (natural illumination) in a greenhouse. For the fluorescence microscopic observation, 200 plantlets were used at each treat. For the SEM observation, 20 plants were used at each treatment. Because this study mainly focus on the movement in the vascular bundle using GFP-expressing $F$. oxysporum f. sp. cubense, and in order to make research results closer to production conditions in the field, the wounded-root inoculation method was used. In the process of banana production, some roots grow onto the soil and are easily wounded by production operations such as pesticide spraying, fertilization, and watering. The roots of banana plants were cut approximately 0.5 to $1 \mathrm{~cm}$ from the root tip, dipped into the $F$. oxysporum f. sp. cubense spore solution, and incubated for $2.5 \mathrm{~h}$. For the control plants, their roots were dipped into PDB (mock inoculation). All of those plants were then returned to normal conditions in pots in the greenhouse.

Microscopic observation with a confocal microscope. Following inoculation, the inoculated plants were examined daily during the first month and weekly in the second month. Three plants per time
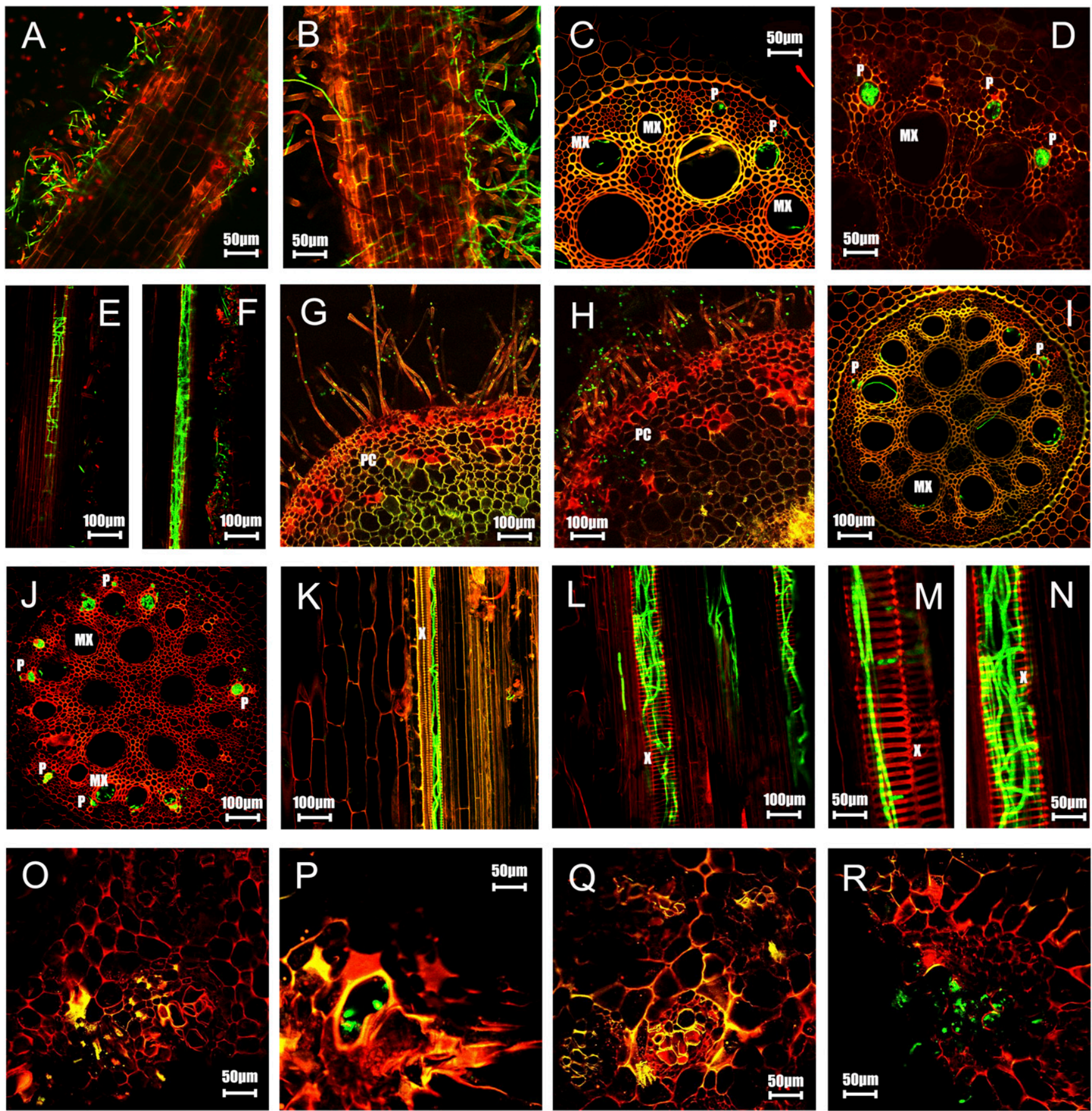

Fig. 1. Examination of the infection process using green fluorescent protein (GFP)-expressing Fusarium oxysporum f. sp. cubense race 1 (Foc1) and race 4 (Foc4). Spores and hyphae of the GFP-expressing A, Foc1 and B, Foc4 attached to the root hairs and root epidermis of banana at 2 days postinoculation (dpi). Cross sections located $5 \mathrm{~mm}$ from the inoculation sites of the banana roots showing the presence of $\mathbf{C}$ and $\mathbf{E}$, Foc1 and $\mathbf{D}$ and $\mathbf{F}$, Foc4 hyphae at 3 dpi. $\mathbf{M X}=$ metaxylem and $\mathbf{P}=$ phloem. Spores of $\mathbf{G}$, Foc1 observed on the root hairs of banana at $7 \mathrm{dpi}$, whereas $\mathrm{H}$, spores of Foc4 were observed on the root hairs as well as in parenchymal cells. $\mathrm{PC}=$ parenchymal cell. Banana root cross sections at 7 dpi showing presence of I, Foc1 and J, Foc4. Few Foc1 hyphae were observed in a few xylems (I) while many more Foc4 hyphae were present in many xylems and phloems (J). Longitudinal sections of banana roots at 7 dpi showing the presence of $K$ and $M$, Foc 1 hyphae and $L$ and $\mathbf{N}$, Foc 4 hyphae in xylems. $X=x y l e m$. 0 to $R, R$ hizome cross sections at 2 months postinoculation. Foc4 ( $P$ and $R$ ) but not Foc1 $(O$ and $Q$ ) hyphae were observed in the vascular tissues of banana rhizomes. 
and five roots per plant were sampled in each treats. But more plants ( 3 to 10 plants) were sampled when found difference between Foc1 and Foc4. For the microscopic examination, banana roots were washed in sterile distilled water and then observed with a laser confocal microscope (OLYMPUS FV10-ASW) using filter blocks to select for spectral emission at $488 \mathrm{~nm}$ (matching the GFP) and $595 \mathrm{~nm}$ (matching root autofluorescence) (Li et al. 2013). The colonization of $F$. oxysporum $\mathrm{f}$. sp. cubense on the surface of roots, in the roots, and in the rhizomes of banana was observed. The number of roots with fungi on the surface, the number of roots with fungi in vascular tissues, the number of vascular bundles with fungi in per infected root, and the number of rhizomes with fungi in vascular tissues were counted (Table 1). Then, 3 days of data were used to do statistical analysis (Table 1). Statistical significance between Foc1 and Foc4 was carried out using Student's $t$ test by SPSS 11.0 software.

Sample preparation for SEM and microscopic observation with SEM. The detached banana roots were fixed with $2.5 \%$ glutaraldehyde solution for 2 to $3 \mathrm{~h}$, then cut into pieces approximately $5 \mathrm{~mm}$ in length with blades, placed into phosphate buffer (0.1 mol-liter $\left.{ }^{-1}, \mathrm{pH} 7.2\right)$, and soaked for approximately $1 \mathrm{~h}$. The soaking step was repeated three to four times. The samples were then dehydrated through a graded series of ethanol $(30,50,70,80,90$, and $100 \%$, once for $20 \mathrm{~min}$ at each step), immersed in a 1:1 mixture of isoamyl acetate and ethanol for $20 \mathrm{~min}$, then immersed in $100 \%$ isoamyl acetate for $20 \mathrm{~min}$. The tissues were dried in a critical point dryer (Emitech $\mathrm{K} 850$; Emitech Ltd.) using liquefied $\mathrm{CO}_{2}$ as the transitional fluid (Pathan et al. 2008). The root tissues were placed on the conducting resin and then observed using a desktop scanning electron microscope (PHENOM; FEI/Phenom-World).

\section{Results}

Observation of the infection process with GFP-tagged $F$. oxysporum f. sp. cubense strains with laser confocal microscopy. Roots from banana plants were cut and dipped into the spore solution of GFP-expressing Foc4 and Foc1. The fluorescence signal was subsequently observed with a laser confocal microscope. The results are shown in Figure 1. Hyphae of both the GFP-expressing Foc1 (Fig. 1A) and Foc4 (Fig. 1B) readily attached to the root hairs and root epidermis of banana at $2 \mathrm{dpi}$, and there was no significant difference between Foc1 and Foc4 (Table 1). At 3 dpi, both Foc1 (Fig. 1C and E) and Foc4 (Fig. 1D and F) invaded the vascular tissues of the banana roots, because hyphae were observed in the vascular tissues approximately $0.5 \mathrm{~cm}$ from the cut sites in the roots. The number of roots and vascular bundle with fungi during 3 days (from 3 to $5 \mathrm{dpi}$ ) were calculated (Table 1). In total, 50 roots of 10 plants were calculated. There were 13 roots ( $26 \%$ of the total 50 roots) with GFP-tagged Foc1 and 17 roots (34\%) with GFP-tagged Foc4 in vascular tissues. In addition, there were significant differences in the numbers of roots invaded by the two $F$. oxysporum f. sp. cubense races from the statistical analysis based on the data of 3 days.

Many differences were found between roots infected by Foc1 and Foc4 at 7 dpi. First, many Foc4 spores were observed on the root hairs and also in the parenchymal cells of banana roots (Fig. 1H), whereas fewer Foc1 spores were found only on the root hairs of banana (Fig. 1G). Second, few Foc1 were observed in a few xylems (Fig. 1I, K, and $\mathrm{M}$ ) while many more Foc4 were present in many xylems and phloems (Fig. 1J, L, and N). The number of vascular bundles with fungi in roots during 3 days (from 7 dpi to $9 \mathrm{dpi}$ ) were calculated. We found that there were about 6 vascular bundles (the mean of 11 roots) with GFP-tagged Foc1 per root (24\% of the total 45 roots) and about 14 vascular bundles (the mean of 25 roots) with GFP-tagged Foc 4 per root (56\% of the total 45 roots) (Table 1). There were significant differences between the two F. oxysporum f. sp. cubense races (Table 1).

Approximately 2 months after inoculation, Foc4 was observed in the vascular tissues of banana rhizomes (Fig. 1P and R), whereas no Foc1 was found in rhizomes (Fig. 10 and Q). We found that there were 11 rhizomes (44\%) with GFP-tagged Foc4 in vascular tissues in a total of 25 plants and 0 rhizomes with GFP-tagged Foc1 in a total of 25 plants (Table 1). At the same time, banana plants inoculated with Foc4 showed symptoms of wilting (Fig. 2A) and browning in the rhizome (Fig. 3A), whereas no wilting or significant browning

Table 1. Numbers and percentages of banana roots or vascular bundles infected by Fusarium oxysporum f. sp. cubense race 1 (Foc1) and race 4 (Foc4)

\begin{tabular}{|c|c|c|c|c|}
\hline \multirow[b]{2}{*}{ Parameters } & \multicolumn{2}{|c|}{ Numbers $(\%)$ inoculated with ${ }^{y}$} & \multirow[b]{2}{*}{ Roots/plants } & \multirow[b]{2}{*}{ Data from ${ }^{z}$} \\
\hline & Foc1 & Foc4 & & \\
\hline Roots with fungi on the surface & $47(94) \mathrm{a}$ & $48(96) \mathrm{a}$ & $50 / 10$ & $2-4$ dpi \\
\hline Roots with fungi in vascular tissues & $13(26) \mathrm{a}$ & $17(34) \mathrm{b}$ & $50 / 10$ & $3-5$ dpi \\
\hline Vascular bundles with fungi per root & 6 (24) (11 roots) a & $14(56)(25$ roots $) b$ & $45 / 9$ & 7-9 dpi \\
\hline Rhizomes with fungi in vascular tissues & $0(0) \mathrm{a}$ & $11(44) \mathrm{b}$ & 25 plants & $61,68,75 \mathrm{dpi}$ \\
\hline
\end{tabular}

y Values followed by different letters indicate significant differences between Foc1 and Foc4 treatments $(P<0.05)$.

${ }^{\mathrm{z}}$ Days postinoculation (dpi).
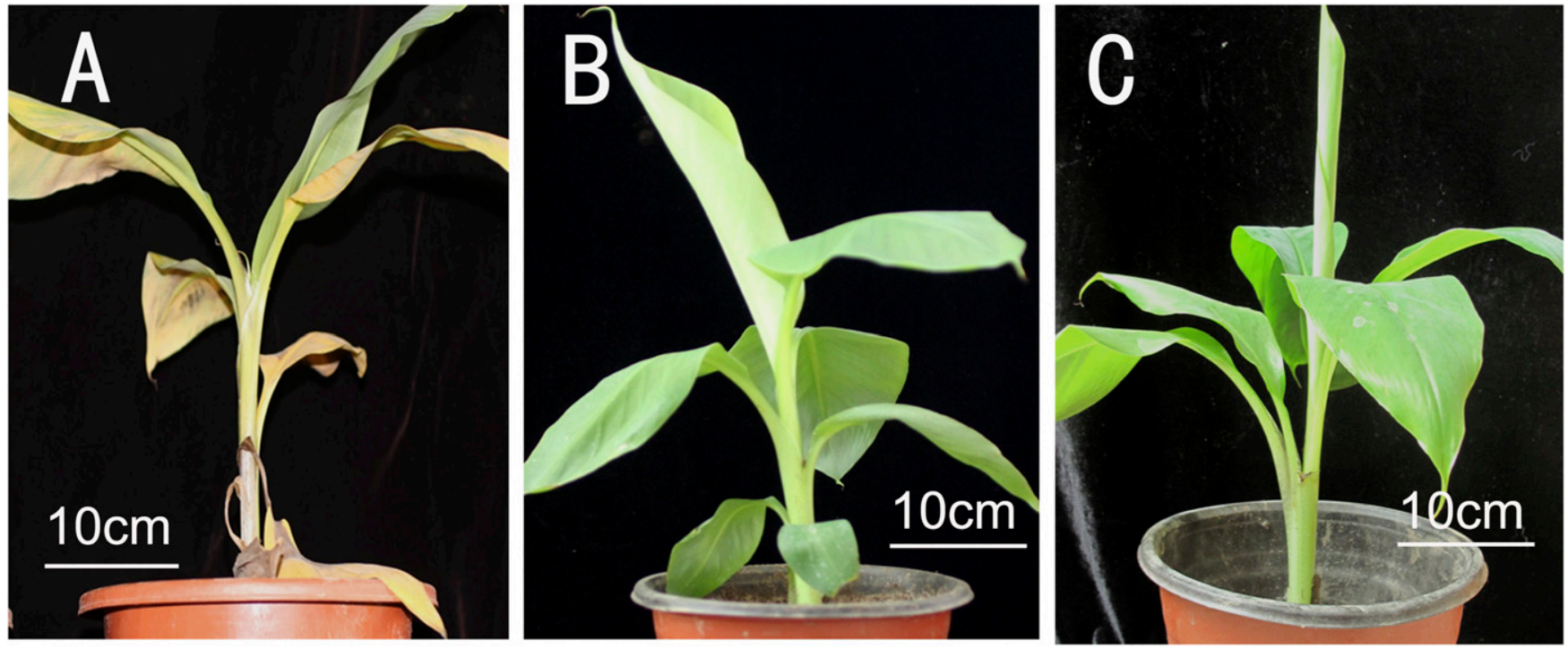

Fig. 2. Phenotypes of banana plants inoculated with A, Fusarium oxysporum f. sp. cubense race 4; B, race 1; or C, water 2 months after inoculation. 
symptoms were found in banana plants inoculated with Foc1 (Figs. $2 \mathrm{~B}$ and $3 \mathrm{~B})$.

Observation of the $F$. oxysporum f. sp. cubense infection process with SEM. To further understand the $F$. oxysporum $\mathrm{f}$. sp. cubense attachment process, we completed observations using SEM. The results are shown in Figure 4 and Table 2. The hyphae and spores of both Foc1 and Foc4 readily attached to the banana roots (Fig. 4A to E) at 1 dpi. Although many Foc1 (Fig. 4G) and Foc4 (Fig. 4H) hyphae were found attached to the cross sections of the cut sites of roots at 2 dpi, many Foc4 hyphae penetrated into banana roots from the intercellular space of the epidermis (Fig. 4L to N; Table 2) but no Foc1 hyphae penetrated into the intercellular space of the epidermis (Fig. 4O; Table 2). Hyphae from both Foc1 (Fig. 4J) and Foc4 (Fig. 4K) were also found penetrating wounded root epidermal cells. Combining these observations with the laser confocal microscopy observations, we conclude that Foc4 can penetrate into banana roots from both the
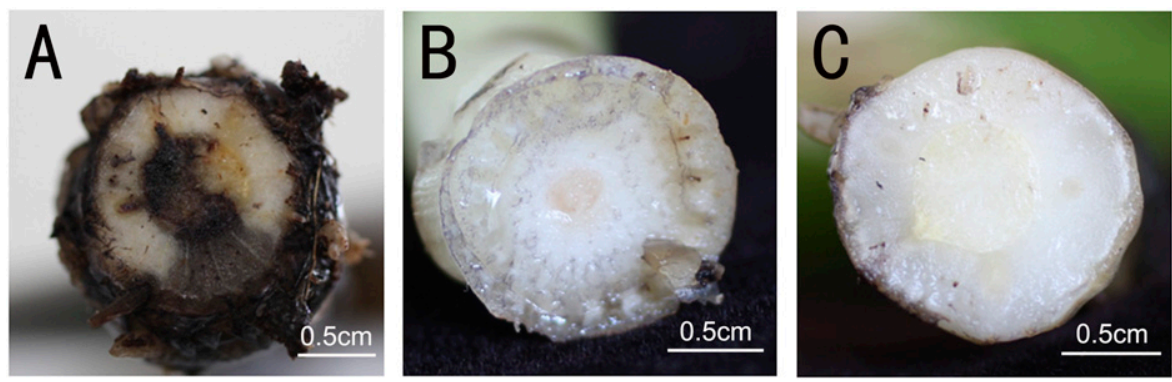

Fig. 3. Different phenotypes exhibited in representative rhizome cross sections from banana plants inoculated with A, Fusarium oxysporum f. sp. cubense race 4; B, race 1; or C, water 2 months after inoculation.
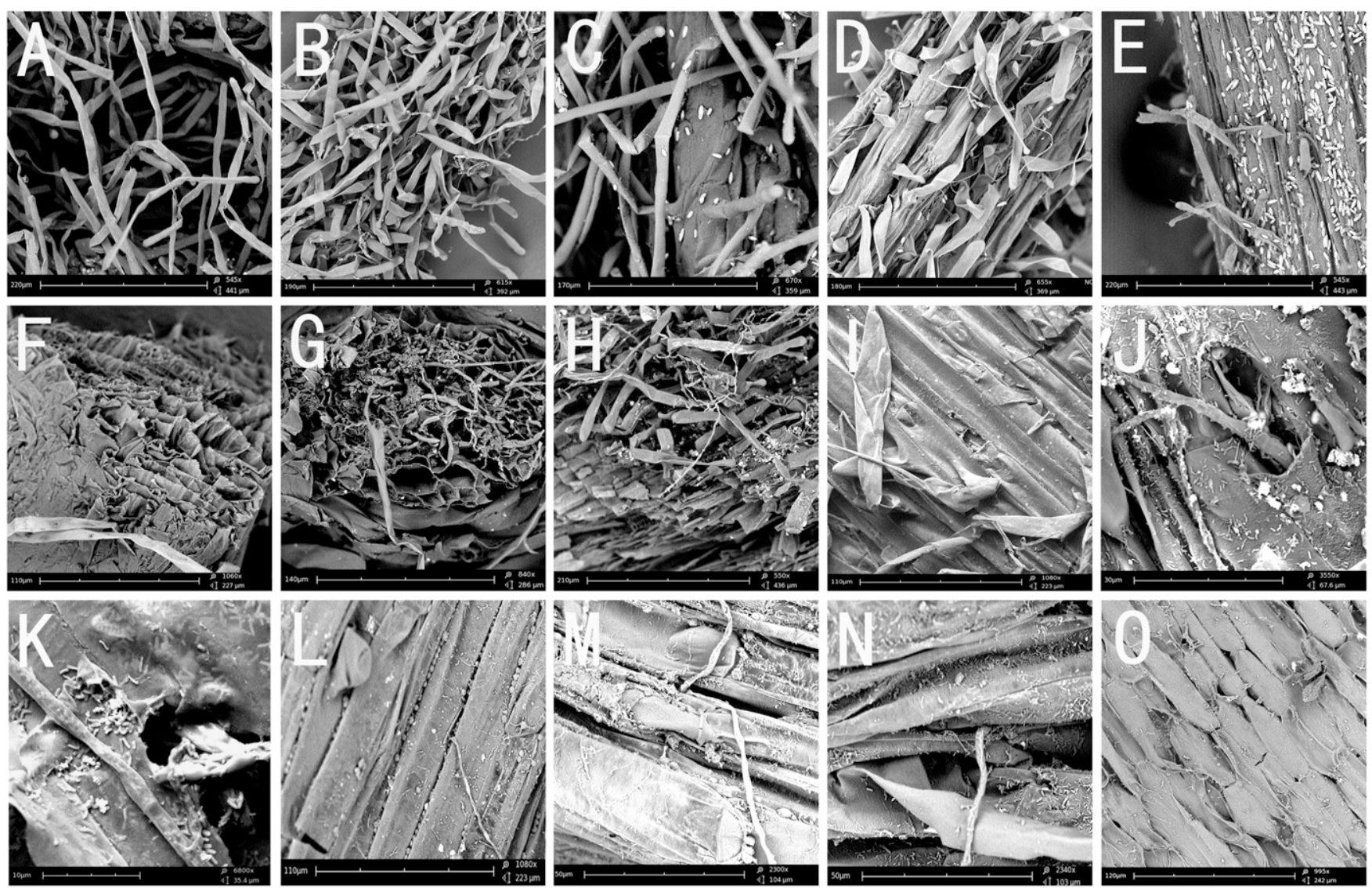

Fig. 4. Comparison of the Fusarium oxysporum f. sp. cubense race 4 (Foc4) and race 1 (Foc1) infection processes with scanning electron microscopy. A, Control without F. oxysporum f. sp. cubense inoculation; B, Foc1 hyphae and C, spores attached to the root of banana at 1 day postinoculation (dpi). D, Foc4 hyphae and E, spores attached to the root of banana at $1 \mathrm{dpi}$. F, Control without $F$. oxysporum f. sp. cubense inoculation; G, Foc1 and H, Foc4 hyphae attached to the cut sites at 2 dpi. I, Control without F. oxysporum f. sp. cubense inoculation; both J, Foc1 and K, Foc4 hyphae were found in the wounded root epidermal cells at 2 dpi. L, M, and $\mathbf{N}$, Many Foc4 hyphae invaded into banana roots from the intercellular space of the epidermis but $\mathbf{0}$, Foc1 hyphae did not.

Table 2. Statistics for Fusarium oxysporum f. sp. cubense race 1 (Foc1) and race 4 (Foc4) penetration from the surface of roots

\begin{tabular}{lccccc}
\hline Race & Penetration between cells & Wound penetration & No penetration & Total view & Number of plants/roots \\
\hline Foc1 & 0 & 5 & 93 & 98 & $10 / 50$ \\
Foc4 & 39 & 3 & 61 & 103 & $10 / 50$ \\
\hline
\end{tabular}


intercellular space of the epidermis and wounds (or cuts), whereas Foc1 mainly penetrated from the wounds (or cuts) but not from the intercellular space of epidermis.

\section{Discussion}

Before the 1960s, Gros Michel was the principal sweet banana variety. However, the worldwide outbreak of the banana Fusarium wilt disease caused by Foc1 led to the worldwide destruction of banana crops in the 1940s to 1960s. The Cavendish subgroup was later found to be more resistant to Foc1 and replaced Gros Michel as the most widely distributed banana cultivar (Price 1995). However, virulent strains of Foc4, particularly FocTR4, to which Cavendish is susceptible have rapidly spread in banana production areas and resulted in tremendous losses in Asia and Australia (Hwang and Ko 2004). Brazil banana, used in this study, belongs to the Cavendish subgroup, which is also resistant to Foc1 but susceptible to Foc4. The infection of banana roots by $F$. oxysporum f. sp. cubense has been studied previously (Beckman et al. 1961; Li et al. 2011; Sequeira et al. 1958; Stover and Waite 1954; Wardlaw 1930; Xiao et al. 2013). It was found that Foc4 can invade plants at or near the root-cap of roots (Stover 1957) and penetrate banana roots through openings in the cell wall and wounds (Li et al. 2011; Sequeira et al. 1958). The fungus then rapidly spreads from one cell to another cell through pores in the cell wall and reproduces within cells ( $\mathrm{Li}$ et al. 2011). Once the fungus reaches the main root base inside the lateral roots, it is well established and will eventually cause successful infection of the rhizome (Rishbeth 1955).

In this study, we found that both Foc4 and Foc1 colonized the root tissues of banana. Li et al. (2011) also found that $F$. oxysporum $\mathrm{f}$. sp. cubense could colonize the root tissues of resistant or tolerant banana cultivars. However, Foc4 was able to penetrate the intercellular space and the root epidermis cell walls of Brazil Cavendish whereas Foc1 were unable to, although Foc1 hyphae were found in root vascular bundles. It is likely that Foc1 penetrates the root of Brazil Cavendish mainly from wounds (or cuts) but not from the intercellular space of the epidermis. This may be one of the reasons why Brazil Cavendish is resistant to Foc1 but susceptible to Foc4.

Our study indicates that both Foc1 and Foc4 are able to spread in the vascular tissues of banana roots in the early infection process. However, more hyphae were in the vascular tissues of the Foc4infected roots than the Foc1-infected roots, although it is difficult to make a precise quantitation of the amounts of hyphae. Foc4 successively penetrated into the epidermis, parenchyma cells, and vascular tissues of roots, whereas Foc 1 was not able to penetrate into the epidermis or parenchyma cells of resistant Brazil Cavendish banana; however, it could penetrate into the vascular tissue of roots from the wounds or cut sites. Furthermore, Foc 4 was observed in the vascular tissues of the rhizome, whereas no Foc1 was found in the rhizome 2 months postinoculation. It was shown that rhizome penetration is the most important step because, once the fungus reaches the rhizome, it is a well-established infection and will eventually result in wilt (Rishbeth 1955). In this study, we did not find Foc4 or Foc1 in the pseudostem of banana during the first 2.5 months after inoculation, although Xiao et al. (2013) reported that Foc4 hyphae spread up to the rhizome and pseudostem xylem by $17 \mathrm{dpi}$. It is possible that the Foc4 isolated from Hainan Island (the tropical area) behaves differently from the Foc4 isolated from the Fujian region (the subtropical or temperate area) of China in the previous study. Additionally, it is also possible that banana plants have different growth performance at different growth stages or different illumination intensities.

Li et al. (2011) also used GFP-tagged Foc4 to study the infection of banana. They found that Foc4 was capable of invading the epidermal cells of banana roots directly, and spores were produced in the root system and rhizome. Roots of Foc4-susceptible 'Guangfen ${ }^{\#} 1$ ' banana plants were well colonized with Foc4 but not those of Foc4-resistant 'Brazilian'. Moderately resistant cultivars reduced spore germination and hyphal growth, whereas the susceptible cultivars did not affect fungal germination and growth. These results are similar to our study. However, the difference are that Li et al. (2011) used different cultivars with only one race Foc4 and focused on the root colonization in different epidermal cells of banana, whereas this research used two races (Foc1 and Foc4) with one cultivar (Brazil, the most popular cultivar in Asia) and focused on $F$. oxysporum f. sp. cubense movement in the vascular bundle and penetration into the root surface. F. oxysporum f. sp. cubense movement in the vascular bundle is very important to banana wilt. It is well known that banana wilt is mainly caused by the hindering of nutrients and water transport through the vascular bundle. Therefore, this study can clarify the whole process of banana infection by $F$. oxysporum f. sp. cubense.

Plants uptake nutrients and water through the vascular bundle. Foc 4 can penetrate into and fill the xylem and air space of Brazil Cavendish roots and then move into rhizome vascular bundles. Once the fungus reaches the rhizome, it interferes with nutrient uptake and upward water transport to the pseudostem and leaves and eventually results in wilting or death. Foc1 could not spread into the rhizome of Brazil Cavendish and nutrient and water transport were not affected; consequently, banana plants can tolerate Foc1 infection. Although some root vascular bundles were infected by Foc1, the level of infection apparently does not affect plant growth and does not result in wilting. Although $F$. oxysporum f. sp. cubense was difficult to eradicate from the soil and control using fungicides (Daly and Walduck 2006), the results from this study suggest that $F$. oxysporum $\mathrm{f}$. sp. cubense infection may be delayed or controlled if we can effectively prevent it from entering into the rhizome or control fungal propagation in the rhizome.

\section{Acknowledgments}

This work was supported by the National Natural Science Foundation of China (number 31271997), the Special Fund for Agro-scientific Research in the Public Interest of China (number 201503110), and the Special Project for the Key Technical Research and Demonstration of Arable Land Improvement in Hainan (HNGDxf2015). We thank Z. Xiong for his assistance during the writing and R. Xiao for kindly providing the pCT74 vector.

\section{Literature Cited}

Beckman, C. H., Mace, M. E., Halmos, S., and McGahan, M. W. 1961. Physical barriers associated with resistance in Fusarium wilt of bananas. Phytopathology 51:507-515.

Daly, A., and Walduck, G. 2006. Fusarium wilt of bananas (Panama Disease) Agnote I51:1-5.

Getha, K., and Vikineswary, S. 2002. Antagonistic effects of Streptomyces violaceus niger strain G10 on Fusarium oxysporum f.sp. cubense race 4: indirect evidence for the role of antibiosis in the antagonistic process. J. Ind. Microbiol. Biotechnol. 28:303-310.

Heslop-Harrison, J. S., and Schwarzacher, T. 2007. Domestication, genomics and the future for banana. Ann. Bot. (Lond.) 100:1073-1084.

Huang, D., Peng, M., and Li, C. 2014. Transformation of GFP gene of several Fusarium oxysporum strains. Chin. J. Trop. Agric. 34:59-62.

Hwang, S. C., and Ko, W. H. 2004. Cavendish banana cultivars resistant to Fusarium wilt acquired through somaclonal variation in Taiwan. Plant Dis. 88:580-588.

Kurtz, A., and Schouten, A. 2009. Shifts in banana root exudate profiles after colonization with the nonpathogenic Fusarium oxysporum strain Fo162. Commun. Agric. Appl. Biol. Sci. 74:547-558.

Li, C., Chen, S., Zuo, C., Sun, Q., Ye, Q., Yi, G., and Huang, B. 2011. The use of GFP-transformed isolates to study infection of banana with Fusarium oxysporum f. sp. cubense race 4. Eur. J. Plant Pathol. 131:327-340.

Li, C., Shao, J., Wang, Y., Li, W., Guo, D., Yan, B., Xia, Y., and Peng, M. 2013 Analysis of banana transcriptome and global gene expression profiles in banana roots in response to infection by race 1 and tropical race 4 of Fusarium oxysporum f. sp. cubense. BMC Genomics 14:851.

Li, M., Xi, P., Jiang, Z., and Qi, P. 2007. Race identification of Fusarium oxysporum f. sp. cubense, the causal agent of banana Fusarium wilt in Guangdong province. Hua Nan Nong Ye Da Xue Xue Bao 28:38-41.

Lorang, J. M., Tuori, R. P., Martinez, J. P., Sawyer, T. L., Redman, R. S., Rollins, J. A., Wolpert, T. J., Johnson, K. B., Rodriguez, R. J., Dickman, M. B., and Ciuffetti, L. M. 2001. Green fluorescent protein is lighting up fungal biology. Appl. Environ. Microbiol. 67:1987-1994.

Pathan, A. K., Bond, J., and Gaskin, R. E. 2008. Sample preparation for scanning electron microscopy of plant surfaces-Horses for courses. Micron 39: 1049-1061.

Pearce, F. 2003. Going bananas. New Sci. 177:26-29.

Ploetz, R. C., and Pegg, K. G. 2000. Fungal diseases of the root, corm and pseudostem: Fusarium wilt. Pages 143-159 in: Diseases of Banana, Abaca and Enset. D. R. Jones, ed. CABI, Wallingford, UK. 
Price, N. S. 1995. The origin and development of banana and plantain cultivation. Pages 1-13 in: Bananas and Plantains. S. Gowen, ed. Chapman and Hall, London.

Rishbeth, J. 1955. Fusarium wilt of bananas in Jamaica. I. Some observations on the epidemiology of the disease. Ann. Bot. 19:293-328.

Sequeira, L., Steeves, T. A., Steeves, M. M., and Riedhart, J. J. 1958. Role of root injury in Panama disease infections. Nature 182:309-311.

Simmonds, N. W., and Sheperd, K. 1955. The taxonomy and origins of the cultivated bananas. Bot. J. Linn. Soc. 55:302-312.

Stover, R. H. 1962. Fusarial Wilt (Panama Disease) of Bananas and other Musa species. Commonwealth Mycological Institute, Kew, UK.
Stover, R. H. 1957. Ecology and pathogenicity studies with two widely distributed types of Fusarium oxysporum f. sp. cubense. Phytopathology 47:535.

Stover, R. H., and Waite, B. H. 1954. Colonization of banana roots by Fusarium oxysporum f. cubense and other soil fungi. Phytopathology 44: 700-701.

Wardlaw, C. W. 1930. The biology of banana wilt (Panama disease). III. An examination of sucker infection through root bases. Ann. Bot. (Lond.) 45: 381-399.

Xiao, R. F., Zhu, Y. J., Li, Y. D., and Liu, B. 2013. Studies on vascular infection of Fusarium oxysporum $\mathrm{f}$. sp. cubense race 4 in banana by field survey and green fluorescent protein reporter. ESci J. Plant Pathol. 2:44-51. 